\title{
Características del último primer día de clases: un nuevo rito de paso entre los estudiantes que finalizan la escuela secundaria
}

\author{
Characteristics of the last first day of classes: a new rite of passage among \\ students who finish high school
}

\author{
Dr. Alfredo Eymann ${ }^{a}$, Dra. Sara Vainman ${ }^{a}$, Dra. Cristina Catsicaris ${ }^{a}$, Dra. Mariana Krauss ${ }^{a}$ y \\ Dra. Valeria Mulli
}

\section{RESUMEN}

Introducción. Los estudiantes del último año de la escuela secundaria suelen realizar una celebración la noche previa al último primer día de clases (UPD).

Objetivos. Describir las características delUPDenuna población de adolescentes que concurrieron a un hospital universitario de comunidad.

Población y métodos. Estudio de corte transversal. Fueron incluidos adolescentes que consultaron en 2018 y participaron del UPD. Se utilizó una encuesta semiestructurada y se solicitó describir narrativamente el UPD.

Resultados. Se analizaron 102 encuestas. Los UPD se realizaron en un lugar privado en el $46,1 \%$; hubo un adulto acompañante en el $58 \%$; consumió alcohol el 78,4\%; ocurrieron lesiones no intencionales en el $9,8 \%$; tuvo problemas con adultos el $44,1 \%$, y volvería a realizarlo el $88 \%$.

Conclusiones. La mayoría consumió alcohol y cerca de la mitad tuvo problemas con adultos. La mayoría de los estudiantes estuvieron conformes y volverían a realizarlo.

Palabras clave: adolescente, conducta de riesgo, rituales, hábitos.

\begin{abstract}
Introduction. The students of the last year of high school usually hold a celebration the night before the last first day of school (LFD).

Objectives. To describe the LFD characteristics in a population of adolescents who attend a community university hospital. Population and methods. Cross-sectional study. Teenagers who consulted in 2018 and participated in the LFD were included. A semi-structured survey was used and it was requested to narratively describe the LFD.

Results. 102 surveys were analyzed; $46.1 \%$ of the LFD were performed in a private place, there was an adult company in $58 \%$, the alcohol consumption was $78.4 \%$, unintentional injuries occurred in $9.8 \%, 44.1 \%$ had problems with adults and $88 \%$ would do it again.

Conclusions. The majority consumed alcohol and about half had problems with adults. Most of the students were satisfied and would do it again.
\end{abstract}

Key words: adolescent, risk behavior, rituals, habits.

a. Servicio de Clínica Pediátrica del Hospital Italiano de Buenos Aires, Ciudad de Buenos Aires.

Correspondencia:

Dr. Alfredo Eymann: alfredo.eymann@hospitalitaliano.org.ar

Financiamiento: Ninguno.

Conflicto de intereses: Ninguno que declarar.

Recibido: 8-3-2019

Aceptado: 9-9-2019 http: / / dx.doi.org/10.5546/ aap.2020.68

Cómo citar: Eymann A, Vainman S, Catsicaris C, Krauss M, Mulli V. Características del último primer día de clases: un nuevo rito de paso entre los estudiantes que finalizan la escuela secundaria. Arch Argent Pediatr 2020;118(1):68-72.

\section{INTRODUCCIÓN}

La adolescencia forma parte del ciclo vital humano que se caracteriza por la búsqueda y consolidación de la identidad, el desarrollo de una autonomía creciente y la planificación y concreción de proyectos de vida. ${ }^{1}$ En muchas culturas, la transición de la niñez a la adultez es celebrada mediante ceremonias de naturaleza simbólica que se realizan en ocasiones especiales y siguiendo patrones establecidos por la tradición. Los jóvenes se alejan de la comunidad para enfrentarse a una situación de riesgo y regresan transformados como integrantes plenos de la misma. ${ }^{2-4}$

Los rituales que realizan los adolescentes son fundamentales para la elaboración de las transformaciones y pérdidas por las que atraviesan y la organización de su nueva identidad..$^{2,3}$ La edad y la forma en la que se celebran dependen de la cultura de cada lugar donde las personas viven y crecen. Entre las tradiciones más conocidas en nuestro país, se encuentran el festejo de los quince años en las mujeres, la fiesta de egresados, el viaje de egresados $\mathrm{y}$, más recientemente, el último primer día de clases (UPD).

El UPD es una celebración realizada por los estudiantes del último año de la secundaria que se desarrolla el día previo al inicio de clases, originada hace menos de una década en las provincias de Cuyo y extendida a otras regiones del país debido al efecto multiplicador de las redes sociales. La consigna del encuentro suele ser reunirse y permanecer despiertos durante la noche para conversar, jugar, bailar, consumir alcohol, y, finalmente, ingresar, la mañana siguiente, al colegio haciendo ruido y disfrazados. 
Se han descrito en diferentes países otros rituales de paso propios del último año de la escuela secundaria, tales como la semana de playa en Estados Unidos (beach week) $)^{5}$ y el viaje de egresados en Australia (schoolies) ${ }^{6-9}$ que se caracterizan por ser encuentros grupales sin la presencia de los padres, en los que se suele tomar alcohol. En nuestro país, es frecuente que los estudiantes del último año de la secundaria realicen un viaje de egresados. Un estudio realizado en la Ciudad Autónoma de Buenos Aires describió que la mayoría de los estudiantes habían consumido alcohol y dormido poco durante el viaje y muchos habían tenido lesiones no intencionales. ${ }^{10}$

El UPD ha sido noticia en los diferentes medios de comunicación y es un tema nuevo de preocupación entre los adultos, debido al consumo de alcohol y a los incidentes generados. No se han encontrado estudios acerca del UPD, por lo cual se propone describir sus características en nuestro medio.

\section{OBJETIVOS}

Describir las características del UPD en una población de adolescentes que concurrieron a un hospital universitario de comunidad.

\section{POBLACIÓN Y MÉTODOS}

Se realizó un estudio de corte transversal. La población fueron todos los adolescentes que realizaron el UPD y consultaron entre el $1^{\circ} \mathrm{de}$ marzo y el 30 de junio de 2018. La muestra se obtuvo por conveniencia y se solicitó el consentimiento oral de los participantes.

Se desarrolló una encuesta, por un grupo de tres médicos pediatras especialistas en Adolescencia. Se llevó a cabo una prueba de validez aparente en un grupo de cinco estudiantes y se realizaron ajustes. No se realizaron pruebas de confiabilidad. Los adolescentes completaron la encuesta en forma anónima e individual en la sala de espera al finalizar la consulta para luego ser depositada en un buzón.

Las variables evaluadas fueron género (femenino, masculino, otro), edad, tipo de gestión del colegio (público, privado), sede hospitalaria a la que concurrió (Ciudad Autónoma de Buenos Aires o San Justo, en la provincia de Buenos Aires), lugar del encuentro (público en caso de haberse realizado en un bar, boliche, salón, plaza o calle; privado en caso de haberse realizado en una casa; ambos en el caso de haberse realizado en un lugar privado y en un lugar público), número de compañeros que participó, compañía de adultos, consumo de alcohol y/u otras drogas, consumo episódico excesivo de alcohol, lesiones no intencionales, ruptura de objetos materiales, número de horas de sueño, ingreso al colegio, problemas con padres, compañeros, autoridades del colegio, vecinos, policía u otros y si volvería a realizar el UPD (véase Anexo en formato electrónico).

Se solicitó describir narrativamente el UPD y las dos mejores y peores situaciones que sucedieron durante este. Se definió la variable problemas con adultos cuando hubo problemas con padres, autoridades del colegio, policía, vecinos y / u otros. Se definió la variable consumo de sustancias cuando hubo consumo de alcohol, consumo episódico excesivo y / $\mathrm{u}$ otras drogas.

Las variables cualitativas se agruparon en categorías definidas por dos médicos pediatras especialistas en Adolescencia. Las variables categóricas se describieron en números absolutos y porcentajes, y las variables continuas, en media y desvío estándar. Se utilizó la prueba $\mathrm{de} \mathrm{chi}^{2}$ y se consideró significativo un valor de $\mathrm{p}$ menor de 0,05. El estudio fue aprobado por el Comité de Evaluación de Protocolos de Investigación de nuestra Institución (número 3845).

\section{RESULTADOS}

Todos los adolescentes que fueron invitados a participar completaron la encuesta. Se recibieron 106 encuestas; se rechazaron 4 por encontrarse incompletas y se procesaron para su análisis 102.

La Tabla 1 describe las características de los estudiantes; la Tabla 2, las de los UPD, y la Tabla 3, los problemas que los estudiantes tuvieron con otras personas. No se encontró una asociación estadísticamente significativa entre haber tenido problemas con adultos y género $(\mathrm{p}=0,22)$, tipo de gestión del colegio $(\mathrm{p}=0,30)$, sede hospitalaria a la que consultaron $(\mathrm{p}=0,18)_{\mathrm{o}}$ consumo de sustancias $(p=0,88)$. No se encontró una asociación estadísticamente significativa entre consumo de sustancias y género $(p=0,06)$, tipo de gestión del colegio $(p=0,94)$, sede hospitalaria a la que consultó $(\mathrm{p}=0,82)$ o haber estado acompañado por un adulto $(\mathrm{p}=0,53)$.

En el análisis cualitativo, los adolescentes describieron los UPD como una fiesta previa al UPD, que consistió en reunirse para divertirse con amigos (bailando, escuchando música, haciendo juegos, etc.), no dormir e ingresar al colegio todos juntos haciendo ruido con pirotecnia, instrumentos musicales y/o cantando. Las situaciones más satisfactorias se organizaron en 
las siguientes categorías: 1) compartir con amigos ("Estuvo todo mi curso unido sin discutir"); 2) la previa ("Nos reímos y bailamos juntos"); 3) el momento del ingreso a la escuela ("Festejando y haciendo ruido con bombos, bengalas, banderas y tirando espuma"). Las situaciones menos satisfactorias se agruparon en las siguientes categorías: 1) dormir poco ("El curso entero se durmió en clases"); 2) emborracharse ("Varios amigos quebraron"); 3) situaciones conflictivas

TABLA 1. Características de los estudiantes que realizaron el último primer día de clases

\begin{tabular}{lcccc}
\hline $\mathbf{N}=\mathbf{1 0 2}$ & $\mathbf{n}$ & $\%$ & Media y DE \\
\hline Género & Femenino & 72 & 70,6 & \\
& Masculino & 28 & 29,4 & $16,9 \pm 0,4$ \\
Edad & & & & \\
Gestión del colegio & Pública & 17 & 83,3 & \\
& Privada & 85 & 83,3 \\
Sede hospitalaria & CABA & 85 & 17,7 & \\
& San Justo & 17 & \\
\hline
\end{tabular}

* Ciudad Autónoma de Buenos Aires.

DE: desvío estándar.

TABLA 2. Características del último primer día de clases

\begin{tabular}{|c|c|c|c|c|c|}
\hline $\mathrm{N}=102$ & & $\mathbf{n}$ & $\%$ & Media y DE & Rango \\
\hline \multirow[t]{3}{*}{ Lugar de realización } & Público & 23 & 22,5 & & \\
\hline & Privado & 47 & 46,1 & & \\
\hline & Ambos & 32 & 31,4 & & \\
\hline Número de participantes & & & & $57,2 \pm 65$ & $2-500$ \\
\hline Adulto acompañante & & 59 & 58 & & \\
\hline Consumo de alcohol & & 80 & 78,4 & & \\
\hline Consumo episódico excesivo de alcohol & & 32 & 31,4 & & \\
\hline Consumo de otras drogas & & 12 & 11,8 & & \\
\hline Accidentes & & 10 & 9,8 & & \\
\hline Rotura de objetos & & 17 & 16,7 & & \\
\hline Horas de sueño & & & & $1,2 \pm 2,3$ & \\
\hline Ingreso al colegio & & 97 & 95 & & \\
\hline Volvería a realizarlo & & 90 & 88 & & \\
\hline
\end{tabular}

DE: desvío estándar.

TABla 3. Problemas con otras personas durante el último primer día de clases

\begin{tabular}{lcc}
\hline $\mathbf{N}=\mathbf{1 0 2}$ & $\mathbf{n}$ & $\%$ \\
\hline Problemas con los padres & 15 & 14,7 \\
Problemas con las autoridades del colegio & 14 & 13,8 \\
Problemas con los vecinos & 19 & 18,6 \\
Problemas con la policía & 12 & 11,8 \\
Problemas con otros & 9 & 8,9 \\
Problemas con adultos & 45 & 44,1 \\
Problemas con compañeros & 11 & 10,8 \\
\hline
\end{tabular}


por la ruptura o pérdida de objetos, lesiones no intencionales y peleas o problemas con otros ("El grupo se separó", "Salimos en la tele por cortar la calle y nos suspendieron”, "Nos echaron del lugar que alquilamos por romper vidrios").

\section{DISCUSIÓN}

El UPD es un encuentro del que participan adolescentes del último año de la escuela secundaria, durante el cual pasan sin dormir la noche previa al comienzo del primer día de clases. Es frecuente el consumo de alcohol y que se generen problemas con adultos.

En nuestra muestra, hay una predominancia de mujeres, que no representa la proporción poblacional. ${ }^{11}$ Esto podría estar relacionado con que las mujeres acceden al sistema de salud en una relación $3: 1$ respecto a los varones, debido a las preocupaciones o consultas vinculadas a aspectos ginecológicos y a una mayor percepción de los problemas de salud. ${ }^{12}$

Los ritos de paso son celebraciones que siguen una secuencia determinada, están presentes en casi todas las culturas y ayudan a superar crisis vitales. Los ritos del ciclo de la vida son las contrapartes culturales de las modificaciones biológicas del proceso vital de las personas; se tratan, fundamentalmente, de una reubicación sociocultural. ${ }^{2,4}$

Los adolescentes se desenvuelven en una sociedad que, en los últimos años, ha tenido importantes transformaciones. En este contexto y frente a la necesidad del cambio, son los mismos adolescentes los que crean los ritos de paso, y es una de sus características constitutivas que se expongan a situaciones de riesgo. ${ }^{13}$

Como dice el antropólogo Le Breton: "Las conductas de riesgo son ritos íntimos de contrabando con miras a fabricar un sentido para poder continuar viviendo". ${ }^{13}$ Esto se manifiesta también durante la celebración del UPD, cuando un gran porcentaje de los participantes consume alcohol. Dicho dato coincide con lo relevado por la encuesta nacional de salud escolar, en la que se observó que el 56,8 \% de los estudiantes secundarios habían bebido, al menos, una bebida alcohólica durante un día o más en los últimos 30 días, con consumo más frecuente en los varones. ${ }^{14}$ En nuestro estudio, no se observó una asociación estadística entre el género y el consumo de alcohol; sin embargo, se notó una tendencia a un mayor consumo de alcohol entre los varones.

Asimismo, existen publicaciones acerca de conductas de riesgo durante la celebración de otros ritos de transición propios de esta etapa de la vida. Una investigación realizada en Estados Unidos demostró que un gran porcentaje de las adolescentes que participaron de la semana de playa (beach week) presentaron conductas de riesgo tales como fumar, beber alcohol, consumir drogas o tener relaciones sexuales inseguras y fue más probable que presentaran dichas conductas quienes las tuvieron antes del viaje. ${ }^{5}$ En una encuesta realizada en Australia para conocer las expectativas de los estudiantes sobre las schoolies, varios participantes consideraron que dicho evento era un importante punto de partida para sus nuevas vidas, un momento de transición a la adultez y que el consumo de alcohol formaba parte natural de este proceso. ${ }^{6}$

Si bien, durante los ritos de paso, los jóvenes no suelen estar acompañados por adultos, para así poder enfrentarse a riesgos, cerca de la mitad de los UPD se realizaron en ámbitos privados y los adultos participaron como acompañantes en más de la mitad de los casos. Esto podría deberse a condicionantes sociales urbanos, tales como los requerimientos contractuales de los establecimientos privados, que exigen la presencia de adultos.

La exposición a situaciones de riesgo es constitutiva en los adolescentes y suelen resolverlas por ellos mismos, junto con sus pares o acudiendo a adultos referentes. ${ }^{14}$ Los adultos acompañantes se suelen encontrar en posiciones ambivalentes entre adoptar conductas restrictivas o permisivas según el significado que cada familia le atribuye al concepto de cuidado en una sociedad que está transitando importantes transformaciones y crisis de muchas instituciones modernas, denominada sociedad del riesgo. ${ }^{15}$

Un estudio sobre la prevalencia de hábitos y conductas de riesgo en los adolescentes de la escuela secundaria de la Ciudad Autónoma de Buenos Aires que habían realizado el viaje de egresados observó que, en relación con las conductas de riesgo, el alcohol fue la droga más usada y que, para todas las conductas de riesgo evaluadas, la asociación más significativa fue con el hecho de haber tenido estas conductas antes del viaje. Además, las lesiones no intencionales más graves descritas se debieron a caídas, ${ }^{10}$ al igual que en nuestro estudio, en el que también se describieron lesiones no intencionales por el uso de pirotecnia, el cual es muy frecuente durante la celebración del UPD.

Las pocas horas de sueño pueden explicarse por la definición misma del UPD, ya que 
los adolescentes se reúnen para permanecer despiertos toda la noche antes de ingresar al colegio. La falta de sueño respecto a las necesidades fisiológicas también es característica de otros ritos de transición, como el viaje de egresados. ${ }^{10}$ Además, las horas de la noche suelen ser un momento en que los adolescentes se perciben con un bajo control por parte de los adultos, por lo que pueden explorar su autonomía.

La mayoría de los participantes tuvieron problemas con adultos o con sus pares. Si bien el consumo episódico excesivo de alcohol ha sido asociado a problemas vinculares y peleas, en nuestro estudio, no se ha encontrado una asociación con el consumo de alcohol, probablemente, por el pequeño número de la muestra.

Aunque un gran porcentaje de los adolescentes estuvo conforme con el UPD y destaca como positivo el poder compartir con amigos, un $12 \%$ no lo realizaría nuevamente. Esto podría deberse a una actitud reflexiva de algunos estudiantes hacia las consecuencias negativas del festejo, tales como emborracharse, tener problemas o peleas y tener sueño al otro día.

Van Gennep ha descrito que los ritos de paso incluyen el riesgo como elemento constitutivo. Además, los adolescentes y jóvenes de todas las sociedades suelen recorrer los márgenes de los actos permitidos por la cultura, en los que presumen un riesgo, para explorar y afirmar su autonomía, que es una de las tareas clave en su tránsito hacia la adultez. En este contexto, los adultos tienen responsabilidades ineludibles con los adolescentes que comenzaron con los primeros gestos de la crianza: el amor, la comunicación, la comprensión, la puesta de límites, la contención y los modelos de rol que les ofrecen.

Por tales motivos, la propuesta es construir con los adolescentes un marco en el que ellos puedan ejercer su autonomía, pero con la contención cercana de los adultos para que puedan transitarla sin correr tantos riesgos. Cada comunidad (familia, autoridades escolares y estudiantes) deberá definir sus estrategias para transitar este rito de paso: talleres o encuentros de reflexión durante todo el año previo, disponibilidad de espacios para el UPD consensuados, reglas acerca de qué se puede y qué no se puede consumir, acuerdos y flexibilidad para el ingreso a la escuela, entre otros. ${ }^{16}$
El presente estudio presenta algunas debilidades, tales como el pequeño número de encuestas y que los participantes consultaron a un hospital de comunidad, universitario y de gestión privada que no representaba a la mayoría de los estudiantes secundarios del país. Sin embargo, se considera que es valioso para comenzar a comprender el significado de estos nuevos comportamientos de los adolescentes.

\section{CONCLUSIONES}

La mayoría de los adolescentes consumió alcohol y cerca de la mitad tuvo problemas con adultos. La mayoría de los estudiantes estuvieron conformes con el UPD y lo volverían a realizar.

\section{REFERENCIAS}

1. Gaete V. Desarrollo psicosocial del adolescente. Rev Chil Pediatr. 2015; 86(6):436-43.

2. Brêtas JRS, Moreno RS, Eugenio DS, Sala DCP, et al. Os rituais de passagem segundo adolescentes. Acta Paul Enferm. 2008; 21(3):404-11.

3. Delaney C. Rites of passage in adolescence. Adolescence. 1995; 30(120):891-7.

4. Jáuregui J. La teoría de los ritos de paso en la actualidad. Boletín Oficial del INAH Antropología. 2002; 68:61-95.

5. Schwartz RH, Milteer R, Sheridan MJ, Horner CP. Beach week a high school graduation rite of passage for sun, sand, suds, and sex. Arch Pediatr Adolesc Med. 1999; 153(2):180-3.

6. Pettigrew S, Biagioni N, Jongenelis M. Anticipating and addressing event-specific alcohol consumption among adolescents. BMC Public Health. 2016; 16:661.

7. Pettigrew S, Biagioni N, Daube M, Jones SC, et al. Understanding and addressing the Schoolies phenomenon. Aust N Z J Public Health. 2015; 39(2):135-40.

8. Lubman D, Droste N, Pennay A, Hyder S, et al. High rates of alcohol consumption and related harm at Schoolies week: a portal study. Aust N Z J Publ Health. 2014; 38(6):536-41.

9. Kang M. The health of "emerging adults" in Australia: freedom, risk and rites of passage. Med J Aust. 2014; 201(10):562-3.

10. Eymann A, Busaniche J, Mulli V, Paz M, et al. Estudiantes secundarios y su viaje de egresados: prevalencia de hábitos, conductas de riesgo y enfermedades en los años 1997 y 2004. Arch Argent Pediatr. 2007; 105(1):17-22.

11. Instituto Nacional de Estadística y Censos. Estimaciones y proyecciones de población 2010-2040. Total del país. Ciudad Autónoma de Buenos Aires: INDEC; 2013.

12. Ministerio de Salud de la Nación, Instituto Nacional de Estadísticas y Censos. Tercera encuesta nacional de factores de riesgo para enfermedades no transmisibles. Buenos Aires: INDEC; 2015.

13. Le Breton D. El cuerpo herido: identidades estalladas contemporáneas. Buenos Aires: Topia; 2017.

14. Linetzky B, Morello P, Virgolini M, Ferrante D. Resultados dela primera encuesta nacional de salud escolar. Argentina, 2007. Arch Argent Pediatr. 2011; 109(2):111-6.

15. Beck U. La sociedad del riesgo: hacia una nueva modernidad. Barcelona: Paidós; 1998.

16. Van Gennep A. The rites of pasage. Chicago: University of Chicago Press; 1961. 


\section{Anexo}

TE INVITAMOS A RESPONDER ESTA ENCUESTA PARA CONOCER LAS CARACTERÍSTICAS DEL ÚLTIMO PRIMER DÍA DE COMIENZO DE CLASES. ES ANÓNIMA Y CONFIDENCIAL.

Género: Femenino. $\square$ Masculino. $\square$ Otro. $\square \quad$ Edad:___años.

Concurrís a un colegio de gestión: $\quad$ Pública. $\square \quad$ Privada. $\square$

El encuentro de desarrolló en un lugar:

Público (plaza, calle, etc.). $\square \quad$ Privado (casa).

Ambos.

Mencioná cuántas horas dormiste (aproximadamente) durante la noche: horas.

Mencioná cuántos compañeros (aproximadamente) participaron del encuentro: compañeros.

¿Estuvieron acompañados por un adulto (padre, madre)?: Sí.

¿Ingresaste al colegio para cursar tu primer día?:

¿Tomaste alcohol durante el encuentro?:

¿Te emborrachaste durante el encuentro?:

¿Tomaste otro tipo de droga durante el encuentro?:

¿Alguien tuvo un accidente?:

¿Se rompió algún objeto?:

$\begin{array}{ll}\text { Sí. } \square & \text { No. } \square \\ \text { Sí. } \square & \text { No. } \square \\ \text { Sí. } \square & \text { No. } \square \\ \text { Sí. } \square & \text { No. } \square \\ \text { Sí. } \square & \text { No. } \square \\ \text { Sí. } \square & \text { No. } \square \\ \text { Sí. } \square & \text { No. } \square\end{array}$

¿Tuvieron (vos o tus compañeros) algún problema con alguno de los siguientes adultos durante el encuentro (podés marcar más de una opción)?:

Padres. $\square \quad$ Compañeros. $\square \quad$ Autoridades del colegio.

Vecinos. $\square \quad$ Policía. $\square \quad$ Otros. $\square$

Describí brevemente en qué consistió el encuentro.

Mencioná las dos mejores cosas que sucedieron durante el encuentro.

Mencioná las dos peores cosas que sucedieron durante el encuentro.

¿Lo volverías a realizar?:

Sí.

No.

Muchas gracias. 